\title{
Risk stratification of PSA-based screening
}

S ince the publication in 2009 of data indicating a survival benefit of PSA-based screening for prostate cancer, efforts have been directed at optimizing the harms-to-benefit ratio of this strategy. Screening 1,410 men and treating 48 in order to prevent one prostate cancer-related death-as determined during the European Randomized Study of Screening for Prostate Cancer (ERSPC) - entails, in the opinion of most commentators, an unacceptably high rate of overdiagnosis and overtreatment. Hans Lilja, Andrew Vickers and colleagues suggest that risk-based selection of men for screening will dramatically refine this approach to cancer detection.

"Risk stratification is ubiquitous in medicine: we triage in the emergency room, refer difficult cases to tertiary care, use adjuvant therapy for higher stage tumors. But we seem to forget this for screening programs: we make recommendations such as 'all men aged 50-70 should have annual PSA tests.' We believe that risk stratifying prostate cancer screening could retain most of the benefits-in terms of reduced mortality - and avoid much of the harm (in terms of unnecessary screening and overdiagnosis)", asserts Lilja.

So, which men are most likely to develop clinically significant prostate cancer, and should therefore be offered screening? To answer this question, the team used blood plasma samples collected during the Malmo Preventive Project in Sweden between 1974 and 1986. Participants in the study were aged 33-60 years at the time of venipuncture. After 20 years or more, the frozen samples were thawed and PSA levels measured in accordance with WHO calibration standards.

Baseline PSA was strongly associated with subsequent development of advanced prostate cancer (area under the curve 0.75). More than $80 \%$ of men who went on to be diagnosed with aggressive disease had an initial PSA level above the median of $0.63 \mathrm{ng} / \mathrm{ml}$.

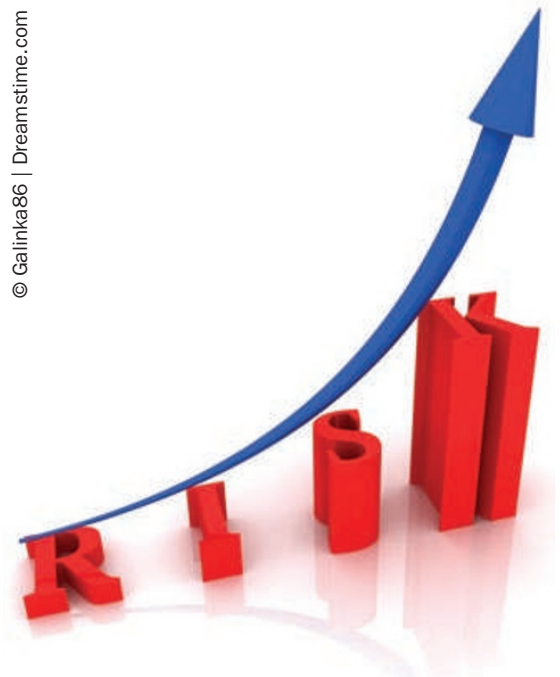

These findings, published in Cancer, are bolstered by the second of the team's recent publications on risk stratification. For their British Medical Journal report, Vickers et al. focused on metastasis and death due to prostate cancer. Again, they showed that a single measurement of PSA-in this case, at age 60 years-is highly predictive of disease spread and mortality during the subsequent quarter of a century.

Lilja explains: "The study in Cancer focused on men aged 44-50 and [shows that PSA] can identify which ... should be advised that particularly close monitoring is essential: over $50 \%$ of the advanced cancers diagnosed in the following 25 years will occur in men in the top 20\% of PSA levels (about $1 \mathrm{ng} / \mathrm{ml}$ or higher). The study in the $B M J$ used a cohort at age 60 , who were followed to age 85 . The risk of death in those with a PSA [level] below the median $(1 \mathrm{ng} / \mathrm{ml})$ was only $0.2 \%$, suggesting that about half of men can be exempted from further screening."

Previous studies by other groups have led to similar conclusions being drawn. Importantly, however, these two new analyses are unique in that the data are virtually free of verification bias. In Sweden, PSA-based screening is uncommon. Indeed, national guidelines advise against screening; most prostatic malignancies are diagnosed clinically. Further confidence in the conclusions comes from the very narrow confidence intervals, the high number of disease cases, and the exceptionally long follow-up.

Lilja believes that "our studies will not only prevent overtreatment and unnecessary screening, but ... actually help save lives. Only about half of men currently get routine PSA tests; imagine how compliance might increase if, instead of saying 'well come back next year, it is what we tell all men', we focused on high risk men and told them 'I don't say this to everyone, but your test shows that you are at high risk. Do make sure you come back to see me in the next year or so"'

Unfortunately, generalizing these recommendations to populations outside Sweden, and to men of other ethnicities, may be problematic. In a third paper, Lilja, Vickers and co-workers report gross disparities between study cohorts with respect to prostate cancer risk at a given PSA concentration. By merging data from eight populations into a single dataset of 25,772 biopsies and 8,503 cancers and applying consistent statistical methods, the researchers identified factors that underlie the discrepancies, including number of biopsy cores, recent screening, and geographical location (USA versus Europe).

Vickers encourages other researchers "to go beyond the culture of competing publications, and work together on shared datasets" in an effort to resolve conflicting findings and optimize the utility of PSA.

Suzanne J. Farley 